\title{
Le webdocumentaire Alma, Voir ou participer
}

The Web Documentary Alma, Seeing or Participating

\section{Bruno Girard}

\section{(2) OpenEdition}

12 Journals

Édition électronique

URL : http://journals.openedition.org/edc/6535

DOI : $10.4000 /$ edc. 6535

ISSN : 2101-0366

Éditeur

Université Lille-3

\section{Édition imprimée}

Date de publication : 1 juin 2016

Pagination : 147-164

ISBN : 978-2-917562-15-4

ISSN : 1270-6841

Référence électronique

Bruno Girard, "Le webdocumentaire Alma, Voir ou participer », Études de communication [En ligne], 46 | 2016, mis en ligne le 01 juin 2018, consulté le 03 mai 2019. URL : http://journals.openedition.org/ edc/6535; DOI : 10.4000/edc.6535 
Le webdocumentaire Alma, Voir ou participer

The Web Documentary Alma, Seeing or Participating

\section{Bruno Girard}

Laboratoire I3M bgirard@unice.fr 
Alma est un webdocumentaire d'Arte au degré de liberté minimal doublé d'un documentaire classique. Dans le webdocumentaire, nous avons le choix de glisser depuis le témoignage d'une jeune femme membre des gangs du Guatemala qui raconte sa propre histoire, vers des illustrations ou des vidéos. Nous nous demanderons pourquoi avoir éprouvé le besoin d'introduire de l'interactivité, là où le documentaire peut suffire? Qu'est-ce qui change lorsque le choix des flux d'images est délégué au lecteur? Que peut nous révéler une interface réduite au seul geste du glissement de la souris (ou du doigt)? D'une manière plus générale, cet article s'interrogera sur les différentes logiques qui s'affrontent au cœur des nouvelles formes d'écritures visuelles.

Mots-clés : interactivité, Alma, sensation, webdocumentaire, récit, attention.
Alma is a web documentary produced by the European culture channel Arte. This interactive documentary, which runs parallel to a traditional film documentary, gives the viewer a minimal degree of freedom. Viewers can choose to move between the testimony of a young female Guatemala gang member who tells her own story, and illustrations or videos. In this article we seek to understand the reasons for introducing interactivity when the traditional documentary form might have sufficed. What happens when the choice of the flow of images is delegated to the viewer? What does an interface reduced to the simple movement of the mouse (or of the finger) reveal to us? More generally, this article examines the confrontation of different strategies within new forms of visual writing.

Keywords: interactivity, Alma, sensation, web documentary, narrative, attention. 


\section{1. \\ Analyse}

Lorsque nous avons choisi de nous intéresser au webdocumentaire Alma, une enfant de la violence, réalisé en 2012, c'était parce que nous pensions avoir trouvé un webdocumentaire à l'interactivité si minimale et en même temps au sujet si fort que nous imaginions pouvoir y découvrir les principes fondamentaux de toute interactivité, mesurer cet écart qui transforme un récit linéaire en toute autre chose dans l'expérience du spectateur. D'autant plus que ce webdocumentaire était doublé d'un documentaire Les Larmes d'Alma réalisé par Ruben Korenfeld lequel a été monté à partir des mêmes rushs que le webdocumentaire sans que l'auteur ait eu à regarder le webdocumentaire.

Le webdocumentaire d'Arte a été réalisé par Isabelle Fougère et Miquel-Dewever Plana, en collaboration avec la société Upian. II a été plusieurs fois primé. Dans le webdocumentaire, nous avons le choix de passer du témoignage d'une jeune femme, Alma, membre d'un gang du Guatemala, le plus souvent filmé de face et en plan rapproché, aux illustrations composées de photographies de Miquel, de vidéo, de dessins et d'animations de Hugh Micol. Il est question de meurtres et de viols.

Le webdocumentaire s'ouvre sur une interface Web au design sobre où l'on retrouve le visage de la jeune femme. Cette interface permet de «démarrer» le webdocumentaire proprement dit ou, au-dessous du pli, de naviguer dans " 4 modules pour comprendre» où sont explicités, sous forme de textes et d'images stylisées, les codes d'appartenance des gangs ou encore l'histoire et la géographie du Guatemala. Cette interface fournit en outre l'ensemble des commentaires qu'a suscités ce document lors de sa diffusion.

Une fois que démarre le webdocumentaire, après un avertissement à propos de la sensibilité des jeunes publics et alors que le visage d'Alma se présente de dos puis de trois quarts, un texte légèrement animé nous informe du principe de navigation: "Lorsque des éléments apparaissent au-dessus d'Alma, vous avez la possibilité d'effectuer un mouvement vers le haut pour accéder à une autre forme de récit. Vous pouvez revenir à tout moment, d'un mouvement vers le bas». Alma apparaît désormais au centre du cadre et entame son récit par un terrible aveu: «j'avais quinze ans quand j'ai voulu entrer dans un gang [...] au début quand je suis arrivée dans cette maison, mes compagnons étaient en train de violer cette fille [...] puis ils m'ont donné l'ordre de la tuer parce qu'elle pouvait nous dénoncer à la Police». Très rapidement, en haut de l'écran apparaît une partie d'une autre vidéo. II suffit de déplacer la souris ou le doigt vers le haut pour que cette seconde vidéo, des enchevêtrements d'habitations sommaires, descende doucement et occupe tout l'écran, tandis que nous entendons toujours la voix d'Alma relatant de quelle manière elle a commis son premier meurtre. Si l'on ne déplace pas la souris vers le bas avant la fin de la seconde vidéo, celle-ci remonte d'elle-même. La navigation est donc 
organisée sur l'axe du temps à partir des différentes étapes du récit d'Alma. Régulièrement, si l'on se maintient sur l'écran des illustrations (terme assez impropre ici pour qualifier la dureté des images présentées) nous sommes ramenés vers Alma. Si les illustrations peuvent parfois déborder sur le flux principal en s'invitant par des animations à l'intérieur même du discours d'Alma, celles-ci respectent rigoureusement l'ordre du récit. Parfois, il n'y a pas d'illustration disponible. C'est le récit d'Alma qui ordonne les illustrations et non l'inverse, c'est la parole d'Alma qui est privilégiée par rapport au documentaire où des scènes additionnelles ont un rôle actif pour la structuration du récit. Ainsi, le documentaire présente Alma dès le départ sur un fauteuil roulant tandis

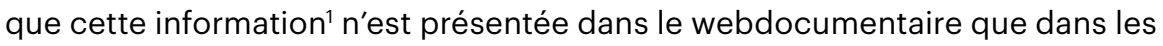
images du générique de fin.

Ceci nous amène à nous demander par quels moyens le webdocumentaire, qui ne donne pas directement les informations sur la situation de la jeune femme ni le contexte particulier des gangs du Guatemala (à moins d'aller les chercher dans les modules additionnels), compte-t-il maintenir l'attention du spectateur? Est-ce le rôle de l'interactivité? Ou est-ce que, dès lors qu'il y a présence d'interactivité, il n'y a plus la nécessité d'une consultation complète? La simple parole d'Alma suffit-elle à maintenir notre intérêt? Plus généralement, est-ce à dire que toutes interactivités procureraient un gain d'attention qui bénéficierait toujours à l'œuvre présentée?

Dans l'attente de pouvoir interroger expérimentalement les deux modalités de réception présentées par ces documents ${ }^{2}$ nous pouvons examiner les commentaires des internautes et des auteurs. Nous pouvons également consulter ce que la littérature dit de l'interactivité, car nous soupçonnons dans la pensée de l'interactivité, la croyance implicite de la supériorité de l'action sur la seule attention.

Ruben Korenfeld, le réalisateur du documentaire ${ }^{3}$, justifie les raisons du traitement distinct appliqué aux mêmes images à partir des différents modes de réception qu'il pressentait suivant que l'on est téléspectateurs ou internautes à partir de deux arguments:

$\times \quad$ Le spectateur peut arriver sur le film, en zappant, il peut le quitter de la même manière, il faut donc «lui donner des raisons de rester".

$\times$ Le documentaire télévisé exige qu'on pose des jalons. Il faut que l'on

1 Dans les modules accompagnant le webdocumentaire, on retrouve aussi une photographie de la jeune femme sur un fauteuil roulant.

2 Nous envisageons de mener une enquête comparative sur la réception du documentaire et du webdocumentaire. Un groupe d'étudiants visionnera le webdocumentaire et un autre groupe visionnera le documentaire, ils devront ensuite répondre à un questionnaire permettant d'évaluer leur implication.

$3 \mathrm{Http}: / /$ television.telerama.fr/television/alma-du-webdocumentaire-au-documentaire-tele, 88994.php. 
puisse comprendre dans quel contexte, dans quel pays a grandi Alma pour comprendre le parcours qu'elle a emprunté. Et puis, cela crée des phases de respiration dans le récit, qui est quand même dérangeant.

Les auteurs du webdocumentaire quant à eux expliquent que "l'internaute est plus acteur que le téléspectateur, on peut davantage le laisser se débrouiller seul ». C'est donc à lui de rechercher le contexte s'il en a besoin. En fin de compte, le webdocumentaire permet paradoxalement de recentrer notre attention sur le témoignage d'Alma. Paradoxalement, parce que l'interactivité se trouve reléguée à la recherche de compléments d'information qui sont ici visuels et sonores. Les réalisateurs des images du webdocumentaire nous dévoilent justement une conception inhabituelle de l'interactivité: "Quand le regard d'Alma devient insupportable, il [l'internaute] peut naviguer et, d'un mouvement du doigt ou de la souris, partir dans l'évocation qu'offre la partie supérieure $[. ..] »^{4}$. Le geste du doigt évacuerait donc l'insupportable, dispenserait de subir la puissance de l'évocation. Propos qui ne manque pas d'être surprenant en regard des images de corps ensanglantés que nous proposent les illustrations. L'interactivité ne serait donc pas au centre du projet.

Une telle position pourtant ne nous éloigne pas de la conception selon laquelle plus nous agirions, plus nous serions attentifs à ce que nous manipulons. Alors que pour un film notre attention ne pourrait être obtenue qu'au prix d'une construction narrative rigoureuse ménageant les attentes des spectateurs, l'interactivité serait ici suffisante pour que nous restions impliqués.

Cédric Mal (Mal, 2013), un journaliste et réalisateur, va plus loin en affirmant que «quand le film de télévision veut tout donner, le webdoc [...] ménage la rétention d'information pour construire un récit plus fort, et bienveillant ». Il ajoute:

× Si le film et le webdocumentaire sont ici constitués par les mêmes ressources, ils ont des effets très différents. Dans le documentaire linéaire, on ne manque rien, et l'on ne manque de rien. Point d'échappatoire possible: on voit tout, et l'on n'a pas le choix. Le webdoc au contraire propose d'accepter de ne pas tout (sa) voir pour justement mieux voir.

Un tel jugement peut-il se généraliser à toute forme d'interactivité? Les limites de l'expérience cinématographique semblent ici posées comme irrémédiables.

Les commentaires qu'Arte a mis en ligne dans l'environnement du projet révèlent en tout cas une distinction manifeste chez les commentateurs entre le sujet lui-même et l'interactivité d'autre part. Si personne ne revendique un plaisir supérieur imputable au mode de navigation entre les images ni ne se plaint de l'interface en quelconque manière, nous avons remarqué que certains 
messages mettaient en avant le thème et la force du sujet: "Le récit est fort, tellement fort que le dispositif multimédia s'efface devant la force du témoignage», tandis que d'autres évoquent le dispositif lui-même: "Au-delà de la dureté des propos, le mécanisme employé est super intéressant». Certains commentaires coïncident avec la diffusion sur Arte du documentaire et font bien sûr alors totalement abstraction de cette dimension interactive. Sur le blog de Cédric Mal, nous voyons un spectateur se plaindre que le débat autour du sujet "s'est concentré presque exclusivement sur le personnage d'Alma, sur son histoire et sa personnalité. Mais presque rien sur ce mode de narration très spécifique et nouveau qu'est le webdocumentaire, pas grand-chose sur les questions d'interactivité ou de support [...]» (Mal, 2013).

C'est donc en raison même de la force du témoignage qu'une interactivité aussi minimale est intéressante à étudier. Ne devrions-nous donc pas nous résoudre à demeurer devant cette jeune femme qui relate les actes monstrueux qu'elle a d'abord subis puis fait subir pour en éprouver toute la violence? L'évocation de ces faits crapuleux et macabres n'était-elle pas suffisante pour stimuler notre imagination, pour en approfondir l'horreur? Il semble bien que le plus proprement dérangeant de ce document réside dans la béance ouverte entre la monstruosité de ce qui est dit et la relative tranquillité qui se dégage de la manière de le dire, du moins tant que cela ne touche pas l'enfant qu'elle a été ou celui qu'elle a perdu. Cette béance n'appartiendrait pas aux illustrations qui finalement nous donneraient des images, certes rudes, que nous pourrions avoir déjà vues ailleurs. Elle appartiendrait en propre à la situation de cette jeune femme et à son attitude. En témoignent quelques commentaires: «...L'ai visionné à l'ancienne, hypnotisé par la violence décrite par la jeune femme en plein repentir sur sa chaise roulante. À 16 ans, dézinguer une femme qui vient de se faire violer devant vous à coups de tournevis et de tessons de bouteille n'invite pas à tapoter sur la tablette».

Pourquoi donc ces illustrations? Pourquoi avoir éprouvé le besoin d'introduire de l'interactivité, là où le documentaire pouvait suffire? Qu'est-ce qui change lorsqu'est délégué (en partie) le choix des flux au lecteur des images? Que vaut dans cette situation la distinction de Gérard Genette entre niveaux narratologiques (Genette, 1972)? À quoi correspondent les éléments fournis dans le webdocumentaire et qu'est ce qui manque et doit être actualisé par le lecteur?

Hanna Arendt (Arendt, 1983: 247), dans la Condition de l'homme moderne, nous rappelle la distinction que le grec ancien entretenait par rapport à l'action. Il y aurait deux formes d'agir, celle par laquelle on commande quelques actions [arkhein] et celle qui conduit à mener une action jusqu'au bout [prattein]. Elle relève en même temps que l'action est considérée comme inférieure à la contemplation dans la philosophie de Platon et des penseurs chrétiens. C'est justement cette distinction, à laquelle nous pourrions rapporter l'action qui consiste à passer d'un niveau à l'autre, celle de la navigation, et l'action 
qui commande de demeurer jusqu'au bout de la sidération devant cette jeune femme et la monstruosité de ses propos, qui doit pouvoir nous permettre d'interroger l'agir lui-même. Il y a d'une part, les actes que cette jeune femme rapporte: comment une jeune femme, après avoir subi violences et outrages pour faire partie d'un gang, est-elle capable à son tour de commettre les pires atrocités? II y a d'autre part nos propres actions concernant ce document: devons-nous naviguer d'un écran à l'autre, ou devons-nous rester figés devant l'innommable? Bien entendu, la nature du choix qui s'offre à nous durant le webdocumentaire est autrement moins difficile que celui qu'Alma doit réaliser pour être acceptée dans le gang: soit celui d'être violée, soit celui d'être battue. Le geste de l'internaute peut paraître ici bien dérisoire quant aux gestes réellement pratiqués par la jeune femme. Mais en même temps, ce geste si anodin ne suscite-t-il pas une interrogation fondamentale sur la manière de conduire un récit : y aurait-il un basculement irrémédiable de notre expérience de spectateur lorsque nous agissons si modestement sur celui-ci?

Déjà, la différence annoncée entre le documentaire et le webdocumentaire est révélatrice d'une conception a priori des modes de réception supposés pour ces deux supports par les auteurs. Au sein même du dispositif, lorsqu'est présenté le mode d'interaction sur l'écran, les auteurs évoquent «une autre forme de récit » comme si le récit devenait différent à partir du moment où l'on usait de la navigation. Voilà, inscrit dans le corps du film lui-même, un présupposé lié à l'interactivité. Conception selon laquelle l'interactivité transformerait le récit lui-même. Conception selon laquelle les dessins, les animations les photographies, les vidéos placés au-dessus du témoignage d'Alma ne se comporteraient donc pas comme un supplément informatif ou comme une simple illustration pour reprendre le terme que nous avons employé jusqu'ici.

2.

\section{Logique du récit}

Jean-Louis Weissberg (Weissberg, 1999: 14) considère l'interactivité comme la source "d'un nouveau régime expressif " sous le vocable d'image actée ${ }^{5}$. Mais qu'en est-il ici du "spectacteur ${ }^{6}$ qui est "l'acteur de son spectacle [...], spectateur des effets de ses actes [...] franchissant sans cesse la barrière sémiotique délimitant l'intérieur (la présentation) et l'extérieur (le dispositif donnant l'accès)» (Weissberg, 2006: 14) quand son geste est aussi élémentaire? Qu'en est-il de cette barrière sémiotique pour la réception elle-même?

5 «L'image actée est une méta-image, portant en elle à la fois sa mise en scène et ses actualisations multiples», op.cit., p.15. Il avait déjà introduit ce terme en1999 dans Présences à distance.

6 Ce terme a été introduit par Réjean Dumouchel en 1991 à propos du disque Laser. Il a été repris ensuite par Jean-Louis Weissberg (1999). 
Lorsqu'on interroge la littérature sur le sujet de l'interactivité, on lui trouve les propriétés suivantes: instanciation dans l'actuel, l'œuvre n'existe que pour autant qu'elle est pratiquée (Weissberg, 2006); métanarration (Di Crosta, 2009), c'est le spectateur qui est le maître d'œuvre de la narration; caractère optatif de I'œuvre (Bianchini, 2012), l'œuvre attend potentiellement une action du lecteur, présence d'une interface; rétroaction possible entre l'utilisateur et le système ${ }^{7}$ (Mahé, 2012). Lev Manovich (Manovich, 2010), le fondateur des software studies, propose de son côté, une autre déclinaison opposant les programmes génératifs à ceux qui sont pré-écrits. Cette distinction est primordiale dans la mesure où elle impacte notre position de spectateur. La possibilité que des images de synthèse puissent être produites en fonction de nos actions, fait de nous un protagoniste autour duquel l'environnement médiatisé va pouvoir lui-même réagir. Ce n'est plus seulement nous-mêmes qui réagissons en regard d'une situation particulière, mais c'est l'environnement qui réagit à notre comportement à travers une interface de plus en plus intuitive. Autrement dit, la dimension ludique et créative est centrée sur nos actions et non plus seulement sur l'univers présenté. Toute intervention à l'intérieur du récit lui-même en change dès lors la portée. L'obligation d'introduire des éléments extradiégétiques par l'intermédiaire d'une interface et celle, pour les spectateurs, d'agir sur cette interface entraînent alors ces dispositifs à rompre avec les mécanismes narratifs inscrits dans la diégèse même (Di Crosta, 2009: 41). Selon Marida Di Crosta, la métalepse, qu'elle définit avec Genette comme pouvant «désigner plusieurs formes de transgression, de l'intrusion du narrateur ou du lecteur dans l'univers de l'histoire à l'irruption d'un personnage dans l'espace de la narration » (Di Crosta, 2009: 39), constitue la figure privilégiée de l'interactivité. La publicité qui interrompt le film que nous regardons n'empêche pas que nous replongions ensuite dans celui-ci, au point où nous l'avions laissé. Il semble que le contexte fictionnel ait été conservé malgré l'interruption inopinée. Au contraire, le choix de l'utilisateur au cours du récit interactif introduit un élément réflexif qui modifie le contexte fictionnel et cognitif de l'œuvre. Le contexte n'est plus le même après notre choix. Ce n'est pas une simple suspension d'un récit au détour d'une interface, mais la transformation de celui-ci et un positionnement cognitif différent de l'utilisateur.

Cependant, personne n'est passif devant quoi que ce soit comme l'évoque Emmanuel Mahé (Mahé, 212: 121) en se référant à Umberto Eco et Hans Jauss. Le lecteur d'un roman qui retourne en arrière pour relire un passage ou encore inscrit sur le bord une réflexion qui lui vient n'est pas plus inactif que celui qui réalise la même chose à partir d'une tablette tactile. Peut-on dire que la lecture n'est pas fragmentée déjà par toutes ces pratiques? Mais ce qui se passe cependant avec les interactions numériques c'est que désormais c'est l'œuvre 
elle-même qui se fragmente et attend une lecture pour être actualisée. Avec les œuvres interactives, c'est à un contenu déstructuré auquel nous avons affaire et qu'on nous charge de reconstruire. Si bien que le récit, celui qui aurait pu être, si nous n'avions pas eu à intervenir, va toujours manquer. L'œuvre se forme au moment même de son utilisation, elle est incomplète toute dans l'incertitude d'une instance qui pourrait être son accomplissement. La nouvelle, Le Livre de sable de Borges (Borges, 1983) décrit un ouvrage qui jamais n'est identique chaque fois qu'on l'ouvre, si bien qu'il est inenvisageable d'en venir à bout. II préfigure la forme que prendront les médias informatisés. Ce qui compte donc avec ce genre d'objet, c'est donc notre expérience de celui-ci.

L'interface du webdocumentaire Alma est plutôt discrète et intuitive, on passe d'un écran à l'autre par le glissement du doigt ou de la souris. Alma ne propose pas un contenu réactualisable c'est-à-dire que notre activité n'implique aucune conséquence sur I'histoire d'Alma. Ce qui s'y déroule s'est déjà passé. Nous pourrions ne jamais intervenir, que le récit se continuerait normalement. Ce n'est donc pas au nom de cette logique-là que ce webdocumentaire est réalisé. II n'y aura pas vraiment de différence d'une consultation à l'autre, si nous entreprenons de recommencer notre lecture de ce document.

3.

\section{Logique de la relation}

A la logique du récit, faudrait-il donc lui substituer la logique de la relation? Emmanuel Mahé dans l'article que nous avons déjà cité, considère qu'il faudrait en terminer avec l'interactivité qui focalise trop la réflexion sur le dispositif technique lui-même et déplacer celle-ci plutôt sur nos pratiques. Nous pouvons parler d'esthétique de la relation à l'image de ce qu'écrit JeanLouis Boissier (Boissier, 2009) lorsqu'il évoque "la relation comme forme " ou encore d'esthétique du flux (Chantosky, 2007), tant il est exact que l'utilisateur va devoir, par les gestes et les décisions qu'il prendra, enchaîner ou connecter des flux de toutes natures. Ainsi l'esthétique de la relation privilégie le geste du spectateur et les projets qui en découlent doivent être pensés d'abord autour du geste spectatorial. Ce qui compte c'est de quelle manière mettre en scène l'agir lui-même en déplaçant l'intérêt de l'œuvre non sur son contenu, mais sur sa pratique. "Le pratiqueur est le dispositif " (Mahé, 2006). L'interface devient alors la partie essentielle d'un dispositif où l'œuvre se constituera dans les actions mêmes des utilisateurs. C'est alors au dispositif de recueillir l'esthétique du projet complet. Parce que nous sommes confrontés à un choix- doit-on naviguer pendant que cette jeune femme nous parle ou doit-on continuer à la regarder?-, ce qui compte n'est alors plus tant notre activité sur l'œuvre que notre relation à l'œuvre et à la situation évoquée par l'œuvre. 


\section{4. Logique de l'interaction}

Nous ne pouvons donc en rester à la seule question de l'interactivité. JeanPaul Fourmentaux (Fourmentaux, 2012: 9) indiquait récemment la nécessité de relancer "l'examen des modalités de la participation du public». Et il est vrai que depuis que Jean-Louis Weissberg avait entrepris cette réflexion sur l'image actée, les innovations technologiques se sont succédées, l'usage de la tablette tactile s'est généralisé, le webdocumentaire, le transmédia, l'économie participative en ligne sont apparus. Si bien que l'interactivité n'est peutêtre plus aujourd'hui qu'une partie d'un ensemble bien plus diversifié qu'il y a quelques années. Comme a pu le suggérer Pierre Morelli ${ }^{8}$, à l'interactivité, il faudrait sans doute désormais préférer l'interaction. Car l'interaction peut désormais se situer en amont du tournage, dès l'écriture d'un projet à travers des protocoles collaboratifs, pendant la diffusion ou après celle-ci. Elle peut être planifiée à partir des nœuds du récit pendant la projection (par exemple: Twixt, 2011, de Francis Ford Coppola); elle peut se manifester à tout moment du récit par des actions des spectateurs (App); elle peut se construire sous une forme générative [données] ou à travers une interface basée sur de l'intelligence artificielle; elle peut se pratiquer à partir d'un second écran en simultané ou encore sur une plateforme Internet, en différé, permettant d'interagir avec les auteurs et acteurs de l'œuvre; elle peut provenir à la limite de la production des spectateurs lorsque ceux-ci détournent ou restituent les images d'un film ou d'une série [fan fiction, found footage, mashup, machinima] (Jenkins, 2013).

Lorsque Henry Jenkins traite de la notion de convergence dans les médias à partir de la question de la participation du consommateur, il insiste sur son mouvement irrémédiable. "Je veux montrer comment la pensée de la convergence est en train de transformer la culture populaire des États-Unis » (Jenkins, 2013: 27). L'interactivité que Henry Jenkins distingue de la participation n'est pas à proprement parler l'objet de sa réflexion, mais elle constitue bien avec les réseaux les conditions nécessaires à notre participation aux médias. Ce ne sont pas des «tuyaux» dont il parle qui permettent d'acheminer sur un même support des contenus diversifiés, mais bien des contenus eux-mêmes et surtout il veut décrire cette attente participative qui anime les consommateurs. L'interactivité est bien une des manières les plus communes d'inclure le spectateur dans le spectacle. Il ne dit pas qu'au film doit succéder le jeu vidéo, mais insiste sur le fait que tous les médias sont concernés; il ne dit pas que cette dimension interactive doit supplanter le régime narratif antérieur, mais il remarque l'apparition d'une forme nouvelle de récit, le «storytelling transmédia ». Il écrit donc:

8 Remarque formulée lors de la $20^{\circ}$ édition du Colloque franco-roumain en information-communication, 30-31 octobre et 1er novembre 2014. 
La plupart des critiques de cinéma apprennent à penser en terme de structures narratives très traditionnelles. Ils parlent de plus en plus d'un effondrement du récit [...] Nous assistons en réalité à l'apparition de nouvelles structures narratives qui créent de la complexité en étendant le champ des possibilités narratives plutôt qu'en poursuivant un chemin unique avec un commencement, un milieu, une fin (Jenkins, 201: 110).

C'est ainsi que David Bordwell (Bordwell, 2009), dans une communication en ligne assez critique sur cette position, ne manque pas de citer Lance Weiler, un réalisateur et designer transmédia qui a travaillé notamment avec Cronenberg sur le projet transmédia Body mind change (2013), lequel affirme que l'explosion de la technologie numérique a transformé le récit que les cinéastes doivent suivre: au lieu de créer un script, avec ses trois actes, le cinéaste devrait produire une bible [à la manière donc des séries], qui trace un ensemble de personnages et d'événements qui se distribue à travers les différents médias, cinéma, Web, mobiles, Réseaux sociaux, jeux, et toutes sortes d'autres platesformes. C'est ainsi que Henry Jenkins aime à rappeler ce que Pierre Levy dans L'Intelligence collective en 1994 avait déjà suggéré «que la distinction entre auteur et lecteur, producteur et spectateur, créateur et interprète va s'effacer » (Jenkins, 2013: 96).

C'est justement de cette position que nous aimerions discuter à travers ce webdocumentaire: il semblerait que la notion de convergence défendue par Henry Jenkins postulerait implicitement que la participation serait plus stimulante que la consommation elle-même et générerait une implication plus grande de la part du spectateur que la simple attention à un programme culturel. Ce qui signifie que l'agir et l'attention appartiendraient à même régime cognitif. Ou pour le dire autrement que les phénomènes nécessitant notre interaction ont notre préférence sur les phénomènes n'impliquant que notre attention. A priori, la dimension cognitive n'est pas explorée par Henry Jenkins qui préfère aborder la notion de convergence à partir de la question des fans en explorant leur désir de démocratie en regard des médias de masse, ce qui n'est pas si nouveau ${ }^{9}$. Tandis que nous voudrions postuler l'idée que les mécanismes cognitifs impliqués dans ces dispositifs sont de natures si différentes qu'ils produisent des comportements - et des plaisirs - réceptifs fort différents. Si bien, parce que c'est bien là la question, que ni la position du conteur-dont Walter Benjamin (Benjamin, 2000:121) constatait déjà le déclin par rapport à la forme de communication qu'est l'information ${ }^{10}$ - ni celle du cinéma

9 Déjà en 1991, Rejean Dumouchel écrivait: "Dans une œuvre interactive, le spectateur quitte la salle et monte sur la scène. II réclame le droit à la participation, l'opportunité de vivre dans le spectacle plutôt que de le regarder sans pouvoir d'intervention. II réclame un nouveau statut de spectateur, il devient acteur, finalement spectacteur ».

10 Walter Benjamin écrivait:«Il s'agit d'un phénomène concomitant à l'évolution historique des forces productives: ce mouvement qui, au cours des siècles et de façon tout à fait progressive, a éliminé le récit du domaine de la parole 
ensuite, ne peuvent être menacées par les structures interactives nouvelles. Ce postulat est déjà perçu de manière critique quand David Bordwell, dans son dialogue avec Henry Jenkins, affirme que «le visionnement de film est déjà une expérience active et participative» (Bordwell, 2009) et surtout lorsqu'il nous rappelle que Lance Weiler en compagnie d'Henry Jenkins a réalisé une liste de degrés d'interactivité dans laquelle le «sit back and watch»- asseyez-vous et regardez-constituerait le niveau le plus bas. Les neurosciences donnent plutôt raison à Bordwell lorsqu'elles parlent de "boucles perception-action » pour qualifier notre lecture itérative d'une image (Lachaux, 2014) qui est toujours partielle, nous permettant de nous focaliser tour à tour sur telle ou telle partie de l'image. Nous ne sommes jamais sans agir devant une image.

La science du cerveau définit l'attention de la manière suivante: «quand la lecture est attentive, ces régions de haut niveau sont envahies par l'activité neuronale, alors qu'elles restent inactives lorsque la lecture est distraite» (Lachaux, 2014), pour ajouter:

$\times \quad$ Au cœur de ce processus, l'attention guide le choix de la perception (en privilégiant une image, une émotion, une image mentale...) et celui de l'action qui lui est associée (qu'il s'agisse d'une action motrice ou «mentale») [...]. C'est pourquoi la qualité de notre attention a tant d'influence sur la qualité de nos actions.

Ce qui signifie, non pas que notre attention augmente avec notre action, mais que nos actions dépendent surtout de notre attention. Selon Jean-Philippe Lachaux", I'interaction à travers "a saillance spéciale» que procure celle-ci augmente pourtant notre concentration sur ce avec quoi nous interagissons, mais elle peut aussi nous détacher du reste en provoquant un effet de cécité cognitive (Simons \& Chabris, 1999). D'un autre côté, par l'intermédiaire des neurones miroirs, il semblerait que la seule vision d'un acte sans la nécessité de le reproduire serait pleinement satisfaisante aussi. Pouvons-nous en conclure que la vision nous laisserait plus libres d'attacher notre attention à des éléments divers tandis que I'interactivité focaliserait celle-ci là où elle intervient? On comprend dès lors pourquoi il est si intéressant, en raison même de la force du sujet, de pouvoir étudier une interactivité aussi minimale que celle d'Alma dont nous avons observé qu'elle ramenait paradoxalement le témoignage de la jeune femme au centre de notre attention.

On pourrait considérer que plus l'internaute est libre de circuler à travers les objets qu'on lui propose, plus il est libre de faire des choix, plus son immersion sera grande à l'intérieur du récit. Le raisonnement contraire postulerait que plus

11 En réponse à un mail que nous lui avons adressé, où nous l'interrogions sur les formes de l'attention avec l'action et la vision, mail du 4 janvier 2016. 
I'utilisateur est invité à agir, plus il est désintéressé à ce qu'on lui raconte par ailleurs (il privilégierait l'action). Pour Dajez (Dajez, 2006), plus l'environnement virtuel répond à nos actions réelles, plus nous adhérons à cet espace simulé comme s'il était réel. D'un autre côté, la composition de l'image au cinéma est parfois déjà construite comme une mise à distance de l'action qui s'y déroule. Nous pouvons prendre comme exemple La Ronde (1950) de Max Ophuls où une séquence se joue au-delà des vitres d'une fenêtre et laisse donc le spectateur hors du champ où l'action se noue. Il est fort probable que les plaisirs cognitifs ne sont certainement pas les mêmes entre agir et voir sans participer et que le cinéma a une expérience intuitive de la question.

Pouvons-nous alors vraiment indexer notre implication par rapport aux degrés d'interaction dont nous disposons? Le cinéma dans sa forme naturelle ne disposerait d'aucun degré d'interaction, le webdocumentaire Alma posséderait un degré supérieur, mais sans comparaison encore avec les jeux vidéo au contenu généré? Dans ce cadre-là, le film tel que nous le connaissons ne serait qu'une instance particulière d'une production à contenu diégétique avec un degré d'interaction réduit, le documentaire Alma ne serait qu'une déclinaison particulière du webdocumentaire. Nous savons qu'il n'en est rien ici et que la structure de ces deux récits a été pensée séparément et qu'il n'est pas possible de réduire le premier à une instance du second. Tout satisfaisant que cela puisse paraître, cette approche ne permet pas de rendre compte de cette distinction entre ces deux puissances d'agir que nous avons évoquées avec Hanna Arendt, sinon en les disposant sur un même axe comme des extrémités opposées.

Il y a dans ce débat quelque chose de celui qui sépara les iconodoules des iconoclastes. Noli me tangere, semble dire l'image de cinéma en opposition avec la spécificité des médias informatisés telle que la désigne Marida Di Crosta. Selon elle, "l'interactivité constitue la propriété esthétique principale spécifique aux médias informatisés» (Di Crosta, 2009:13) et ceci a provoqué un conflit d'ordre créatif pour le cinéma. Marida Di Crosta croit pouvoir constater cette tension entre narration et interactivité au sein même du cinéma. C'est ainsi qu'elle explique le retard pris par le cinéma par rapport à d'autres champs artistiques sur la question de l'interactivité: «Si le numérique et la mise en réseaux ont déjà largement infiltré la filière de production du cinéma, aussi bien en amont qu'en aval de la chaîne, c'est l'interactivité proprement dite, dans sa dimension communicationnelle autant qu'esthétique, qui semble constituer l'obstacle majeur à cette rencontre intermédiale et à son devenir " (Di Crosta, 2009: 11). Pour autant, l'interactivité est bien antérieure à l'arrivée des médias informatisés. En 1967, il y a eu l'expérience du kinoautomat où un animateur permettait au public à travers un vote de choisir entre différentes alternatives ${ }^{12}$. En nous éloignant plus loin dans le temps, nous pouvons retrouver des exemples d'interactivité au théâtre qui remontent au XVIe siècle (Bardiot, 2006: 79), de 
même que le théâtre de marionnettes est peut-être la forme d'interaction qui serait la plus ancienne ${ }^{13}$. Mais il n'est pas moins vrai que l'apport des nouveaux médias a exacerbé cette problématique dans la création audiovisuelle.

Pour ce qui est de l'interaction intradiégétique, si en touchant du doigt un personnage de dos à travers notre tablette tactile celui-ci se retourne, simulant ainsi ce qui se produit dans la vie courante, il est probable que la conséquence de notre geste nous impressionne beaucoup, du moins, les premières fois. Mais en sera-t-il de même avec le temps? S'il faut déplacer un curseur pour augmenter notre vitesse d'action dans un jeu, il est probable que ce ne soit pas essentiel pour notre implication dans celui-ci. C'est pour cela que l'idée récurrente selon laquelle toute activité motrice stimulerait notre adhésion à un récit paraît en soi suspecte, elle ne ferait en réalité qu'accroître notre concentration sur ce que nous faisons. C'est plutôt l'intensité de l'attention cognitive sur certains objets, sur un récit, sur nos actions qui caractériserait notre implication. Autrement dit, être impliqué peut se mesurer logiquement au degré d'inattention qui entoure l'objet sur lequel on se concentre ou sur l'action qu'on est en train d'effectuer. Être impliqué au cinéma, ce serait ne plus voir l'écran, ni la salle, ni les spectateurs, ce serait porter une attention particulière et soutenue sur ce qui se déroule devant nos yeux, être plongé dans l'univers diégétique qu'on nous présente. Encore que l'expérience de chacun montre que notre attention a ses limites et se porte aussi bien sur toutes autres pensées en lien ou non avec les images. Il faudrait donc inverser notre vocabulaire et dire combien notre cerveau est le centre d'une activité neuronale complexe que les images d'un film ne font en réalité qu'exciter par intermittence. II n'est donc pas sûr, d'une part, que l'action tactile soit plus propre à nous impliquer dans un récit que la seule vision et d'autre part que l'on puisse comparer ces activités neurologiques l'une avec l'autre, mais il y a peut-être des modalités particulières de l'une et de l'autre qui favoriseraient notre attention.

\section{5. Logique de la sensation}

Faut-il donc choisir entre voir ou participer? Dilemme que Marida di Crosta relève bien, à la suite de Lev Manovich dans Le Langage des nouveaux médias, pour justifier le déplacement vers un mode alternatif à travers les choix

13 Quelque part, le théâtre des marionnettes et l'ancêtre le plus direct des formes interactives. Dans le livrell Des Histoires d'Hérodote on trouve l'évocation de la manière dont les Égyptiens célébraient Osiris: «au lieu de phallus, les femmes promenaient de village en village des statuettes de la hauteur d'une coudée dont la partie sexuelle, presque égale au reste du corps se mouvait par des ficelles». Il est à noter que le logiciel auteur Director (Adobe-Macromédia) utilisait le terme puppet (pantin) pour nommer les instances des objets visuels manipulables. 
esthétiques de réalisateurs comme David Lynch ou Abel Ferrara par exemple. Il est vrai que ces œuvres marquent une rupture avec une narration linéaire et permettent aux spectateurs de s'interroger sur le dispositif filmique. Elles ouvrent ainsi des «brèches vers l'interactivité » (Di Crosta, 2013: 82).

Marida Di Crosta (Di Crosta, 2013: 21) note donc, fort justement, qu'à partir des années 90 le cinéma cherche par une certaine forme de déconstruction «à entraîner le spectateur au-delà du seuil de l'univers diégétique du film ». Selon elle, ces expériences aspirent à être «plus en adéquation avec la complexité et l'informatisation de l'époque». En même temps, dans la cinématographie de David Lynch, d'Abel Ferrara, et de Philippe Grandrieux, le récit aporétique ou lacunaire, s'il intervient déjà contre une forme de linéarité de la narration que les langages des nouveaux médias ont contribué à dénaturaliser, permet en réalité toute autre chose-Grandrieux interrogé par Antoine de Baecque dans Les Cahiers du cinéma l'énonce explicitement-, il vise la sensation: «la sensation, c'est ce qui se transmet directement, en évitant le détour ou l'ennui d'une histoire à raconter » (Deleuze, 1989). Si le cinéma s'est depuis quelque temps orienté vers une déconstruction narrative, à la manière que décrit Gilles Deleuze à propos du peintre Francis Bacon, c'est surtout pour nous plonger un peu plus loin dans l'éprouvé. Ce qui est vécu dans ces projections ne peut l'être que dans l'abandon, voire le blocage de tout mécanisme réflexif conscient. II faut se livrer aux images qui agissent directement "sur le système nerveux » ainsi que l'exprime Francis Bacon, pour éprouver la sensation. C'est exactement le pendant de l'interactivité, se laisser porter par les images. Il y a bien dans cette optique, basculement du champ symbolique à celui de l'expérience (Di Crosta, 2013: 93). Mais c'est l'expérience de la sensation, et moins celle de l'action, qui est alors cherchée. La modernité du cinéma se situe peut-être plus fondamentalement dans ces tentatives pour approcher la sensation qu'à travers l'interactivité d'un film comme App (2013), qui reste dans le cadre du divertissement, où certains spectateurs vont être appelés sur leur smartphone pendant la projection. App pose sans doute des questions intéressantes sur les évolutions technologiques possibles du dispositif cinématographique, mais ces innovations apparaissent comme extérieures et surajoutées, une forme d'attraction, tandis que la recherche de la sensation demeure en plein attachée au corps même de l'image, à sa constitution et à sa relation avec le fonctionnement propre de notre cerveau. Il convient dès lors de distinguer immersion et sensation. L'une peut être vue comme un dispositif technologique, un appareillage optique et sonore favorisant l'impression d'être parmi les images dont l'idéal implicite repose sur notre projection totale à l'intérieur des images et notre participation à la diégèse, fantasme merveilleusement traité par Woody Allen dans la Rose pourpre du Caire (1985). L'autre doit être envisagée comme une disposition intérieure des images et de la narration favorisant son impact sur notre disposition cognitive dont le modèle au cinéma pourrait être le film Sombre (1998) de Grandrieux. Dans cette situation nous ne sommes pas dans l'image, pas plus qu'elle n'est en nous, mais elle agit sur nous. L'immersion agit 
sur les sens, manipule leur propriété; la sensation intervient sur nos fonctionnements cognitifs, joue avec ceux-ci, perturbe les mécanismes symboliques et de reconnaissance. On ne peut pas privilégier la technologie comme le seul moteur de l'avant-garde artistique, car ce serait oublier que celle-ci peut très bien se déplacer ailleurs. Toutefois, il n'y a pas non plus de freins particuliers à ce que la technologie aide à produire de nouvelles sensations. C'est déjà le cas d'installations expérimentales qui ne visent pas à nous faire réfléchir sur une situation, mais à l'éprouver. Il est fort probable que s'écartant de l'usage de dispositifs technologiques rudimentaires et bénéficiant des progrès de notre connaissance du cerveau, des innovations favoriseront cette logique de la sensation que Deleuze croit reconnaître dans la pratique de Cézanne et du peintre Francis Bacon.

\section{6. Conclusion}

Nous nous sommes demandé s'il fallait vraiment opposer la vision à l'action ou au contraire s'il fallait en apprécier les plaisirs respectifs. Faut-il donc confier aux jeux vidéo et aux installations artistiques le loisir de l'action spectatoriale et laisser au cinéma celui de nous guider à travers un récit? Ne pouvons-nous pas accepter que des plaisirs différents puissent cohabiter? Des produits culturels hybrides, comme le webdocumentaire Alma, invitent en tout cas à se poser la question. Cela reste à démontrer, mais il semblerait que tout nouveau dispositif ne se substitue à un autre que s'il satisfait les mêmes usages à la fois techniques et culturels, tandis que les anciens dispositifs continueront d'exister si les nouveaux ne font que déplacer ces usages. À l'heure du transmédia, éprouver des sensations, être traversé de flux ou approfondir un mode relationnel à l'œuvre sont donc les choix que la modernité culturelle nous offre. Nous devons donc nous interroger sur leurs modalités respectives. Reste qu'il est urgent de revoir à l'aune de l'évolution de nos connaissances sur le cerveau, un certain nombre de notions attachées aux interactions qui ont été formées avant que ces connaissances nous parviennent. 
Bibliographie

Arendt H. (1983). Condition de I'homme moderne, Paris, Calmann-Levy, 1961, éd.1983.

Bardiot C. (2006). «Du dispositif théâtral au chat». In BarbozaP. et Weissberg Jean-Louis (dirs.), L'image actée. Scénarisations numériques, Paris, L'Harmattan, coll. Champs visuels, pp.73-86.

Benjamin W. (2000), "Le conteur». In Fuvres, Tome III, pp.114-151, Paris, Gallimard1936, éd. 2000.

Boissier J.-L. (2009). La relation comme forme, Dijon, Les Presses du réel.

BordwellD. (2009) «Now leaving from platform 1 », In Observations on film art, http://www.davidbordwell.net/blog, août 2009 .

Borges J.-L. (1983). Le Livre de sable, Collection Folio (n¹461), Paris, Gallimard.

Chatelet C. (2013). «Toucher/ cadrer, toucher/monter: des interfaces haptiques pour un spectateur 'amplifié' ». In Entrelacs, URL: http://entrelacs.revues. org/502, consulté le 14 mai 2014.

ChatonskyG. (2007). «Esthétique du flux». In Rue Descartes1, n55, pp.86-99.
De Baecque A. et Thierry J. (1999).

Les Cahiers du Cinéma, n532.

DajezF. (2006). «La figurine interfacée, à propos de l'Odyssée d'Abe». In Weissberg J.-L. (dir.), L'Image actée-Scénarisations numériques, Paris, L'Harmattan.

Deleuze G. (1989), Logique de la sensation, Paris, Seuil.

Di Crosta M. (2009). Entre cinéma et jeux vidéo: l'interface-film: Métanarration et interactivité, Bruxelles, De Boeck.

Fourmentaux J.-P. (2012). L'Ėre post-média: Humanités digitales et cultures numériques, Paris, Éd. Hermann.

DumouchelR. (1991). «Le Spectacteur et le contactile». In Cinémas: revue d'études cinématographiques/ Cinémas: Journal of Film Studies, vol.1, n³, pp.38-60.

Genette G. (1972).

Figures III, Paris, Seuil.

Jenkins H. (2013). La culture de la convergence, Paris, Armand Colin.

Lachaux J.-P. (2014). «Le cerveau attentif: la dynamique de l'attention», http://www.cortex-mag. net/cerveau-attentif-dynamique-lattention/, octobre 2014.

MahéE. (2012). "Les pratiqueurs», In Fourmentraux J.P. (dir.), L'Ére post-média. Arts, Humanités digitales et Cultures numériques, Éd. Hermann, coll. Cultures numériques. 
MalC. (2013). Webdoc: Du potentiel cinématographique d'Alma: http:// cinemadocumentaire.wordpress. com/2013/04/03/webdoc-du-potentiel-cinematographique-dalma/ date de dernière visite: 15 mai 2014 .

Manovich L. (2010). Le Langage des nouveaux médias, Dijon, Les Presses du réel.

Simons D.-J. and Chabris C.-F. (1999). «Gorillas in Our Midst: Sustained Inattentional Blindness for Dynamic Events ». In Perception, September 28, pp.1059-1074.

Weissberg J.-L. (1999). Présences à distance, Paris, L'Harmattan.

Weissberg J.-L. (2006). L'Image actée-Scénarisations numériques, Paris, L'Harmattan. 